\title{
ESTADO ACTUAL DEL CONOCIMIENTO DE HELMINTOS EN AVES SILVESTRES DE CHILE
}

\section{CURRENT STATE OF KNOWLEDGMENTS OF HELMINTHS INCHILEAN WILD BIRDS}

\author{
Ana Hinojosa-Sáez ${ }^{1} \&$ Daniel González-Acuña \\ ${ }^{1}$ Facultad de Ciencias Naturales y Oceanográficas, Departamento de Zoología, Universidad de Concepción. \\ Concepción, Chile. Email: ahinojosa@udec.cl \\ ${ }^{2}$ Facultad de Medicina Veterinaria, Universidad de Concepción, Departamento de Ciencias Pecuarias. Casilla 537, \\ Chillán, Chile. Email: danigonz@udec.cl
}

\section{RESUMEN}

Se revisaron un total de treinta y tres trabajos en helmintos de aves silvestres en Chile. Identificándose 22 familias y 34 géneros de helmintos. Entre las aves hospederas identificadas, los órdenes Chraradriiformes y Pelecaniformes presentan la mayor cantidad de registros. Este trabajo complementa la lista parcial elaborada por Cattan (1995).

Palabras Claves: Helmintos, aves, Chile.

\section{ABSTRACT}

In Chile 33 publications of wild bird helminths were reviewed. 22 families and 34 genues of helminths were identified. Among the host birds, the orders Charadriiformes and Pelecaniformes were the most frequently reported. This study complements the partial list elaborated by Cattan (1995).

KeYwords: Helminths, birds, Chile.

\section{INTRODUCCION}

Si bien los animales silvestres están generalmente infectados por varias especies de parásitos, rara vez sufren muertes masivas o epizootias, debido a la dispersión natural y territorialismo de la mayor parte de las especies (Schmidt \& Roberts 1984). El parasitismo es fundamentalmente una asociación ecológica, que puede definirse como una relación entre dos organismos (hospedador y parásito) en la que el parásito es dependiente metabólicamente de su hospedador (Melhorn \& Piekarski 1998). Esta forma de vida representa más de la mitad de toda la diversidad animal, lo que se debe en gran parte a la especificidad de cada parásito frente a cada hospedador (Tompkins \& Clayton 1999). A lo lar- go del tiempo, tanto el hospedador como el parásito evolucionan, pudiendo hacerlo en forma paralela (co-evolución) o no (Schmidt \& Roberts 1984). Desde el punto de vista parasitológico, la coevolución es la relación histórica existente entre el hospedador y el parásito, la cual frecuentemente es muy pronunciada (Pérez-Iñigo 1976; Brook 1985). Este concepto es útil para determinar relaciones filogenéticas del hospedador. Cuando se presentan problemas para determinar relaciones de parentesco entre géneros o especies de hospedadores, se puede resolver comparando sus parásitos. En la mayoría de los casos entre más cercanos sean sus parásitos, más cercano serán sus hospedadores (Schmidt \& Roberts 1984).

La mayor parte de la información e investigaciones 
parasitológicas en animales silvestres está concentrada en países del hemisferio norte, especialmente Norteamérica y Europa (Chowdhury \& Aguirre 2001). En Chile, la mayor parte de los estudios parasitológicos han sido realizados en aves de importancia veterinaria, siendo los estudios de helmintos en aves silvestres escasos y esporádicos, sujetos generalmente a eventuales hallazgos.

La biodiversidad es la variedad y variabilidad de los seres vivos y de los complejos ecológicos que ellos integran. La misma se organiza en tres niveles, abarcando los distintos ecosistemas, especies y genes, y su abundancia relativa (Monrrone \& Coscarón 1998). Debido a la necesidad de conocer la naturaleza y magnitud de la biodiversidad de nuestro país, junto a la posibilidad de sentar bases científicamente adecuadas para el desarrollo de estudios de enfermedades en aves silvestres y para el control racional de las especies endémicas de Chile, se persigue confeccionar una lista actualizada de los helmintos parásitos registrados en aves silvestres chilenas.

\section{MATERIALES Y METODOS}

Se realiza una revisión completa de todos aquellos estudios realizados en aves silvestres presentes en Chile publicados en revistas de especialidad, congresos científicos y/o tesis de grado. En el texto son presentados los datos en orden alfabético según la clase y la familia del parásito identificado.

\section{RESULTADOS}

\section{Clase Acantocephala Rudolphi 1808}

En ocho especies de aves se ha identificado sólo una familia, con dos géneros y tres especies.

Dentro de la familia Polymorphidae Meyer 1931 se han registrado los géneros Profilicollis Meyer 1931 y Corynosoma Lühe 1904, ambos provocan daños considerables en el intestino de las aves, tales como destrucción de las vellosidades y criptas glandulares. Es así como, Corynosoma puede comprometer la capa muscular intestinal y Profilicollis hasta la serosa intestinal en infecciones severas (Torres et al. 1993). En cuanto al ciclo de estos parásitos, típicamente incluye a crustáceos como hospedadores intermediarios en ciclos acuáticos y artrópodos en el caso de ciclos terrestres. En algunos casos, son utilizados peces como hospedadores paraténicos (Brockerhoff \& Smales 2002).

El género Corynosoma ha sido registrado en Phalacrocorax brasilianus Gmelin 1789 en el lago Huillinco (42³8'S-7400'O) (Torres et al. 1991a) y en Larus dominicanus Lichtenstein 1823 en el lago Yelcho (4316’S-72¹5’O) (Torres et al. 1992). Más tarde, la especie Corynosoma arctocephali Zdzitowiecki 1984 es identificada en P. brasilianus en los lagos Yelcho, Tagua-Tagua (4139'S$\left.72^{\circ} 09^{\prime} \mathrm{O}\right)$ (Torres et al. 1992), Llanquihue (41 ${ }^{\circ} 04^{\prime} \mathrm{S}$ $72^{\circ} 48^{\prime}$ O) (Torres et al. 1993) y en ejemplares de Phalacrocorax atriceps King 1828 del lago TaguaTagua (Torres et al. 1992).

Paralelamente, el género Profilicollis fue aislado por Torres et al. (1993) en ejemplares de Larus maculipennis Lichtenstein 1823 provenientes de los

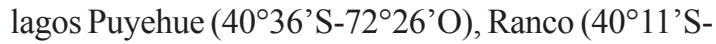
$\left.72^{\circ} 22^{\prime} \mathrm{O}\right)$ y Maihue $\left(40^{\circ} 15^{\prime} \mathrm{S}-72^{\circ} 02^{\prime} \mathrm{O}\right)$ y en Nycticorax nycticorax (Linneus 1758) del lago Ranco. Zamorano et al. (1999) identifican este género en ejemplares de L. dominicanus de las costas de Coquimbo (29 $\left.57^{\prime} \mathrm{S}-71^{\circ} 22^{\prime} \mathrm{O}\right)$.

Por otro lado, la especie Profilicollis antarcticus Zdzitowiecki 1985 fue aislada de L. dominicanus en la costa Yaldad $\left(43^{\circ} 07^{\prime} \mathrm{S}-73^{\circ} 44^{\prime} \mathrm{O}\right)$, los lagos Huillinco (Torres et al. 1991a) y Yelcho (Torres et al. 1992) y de $P$. atriceps provenientes del lago Tagua-Tagua (Torres et al. 1992).

Por último, Oliva et al. (1992) reportan a Profilicollis bullocki Mateo, Córdova \& Guzmán, 1982 desde Larus modestus Tschudii, 1843 y Larus serranus Tschudii, 1844, así como de playeros del género Calidris Merrem, 1804 en las costas de Antofagasta $\left(23^{\circ} 29^{\prime} \mathrm{S}-70^{\circ} 25^{\prime} \mathrm{O}\right)$ en el norte de Chile.

\section{Clase Cestoda Rudolphi 1808}

En 17 especies de aves se ha identificado un total de siete familias de céstodos, con diez géneros y 13 especies.

Aporina delafondi Railliet 1892 (Sin.: Killigrewia delafondi) céstodo cosmopolita, único representante de la familia Anoplocephalidae Cholodkovsky 1902 descrito para colúmbidos, ha sido registrado en Chile en ejemplares de Columba livia Gmelin 1789 en Santiago $\left(33^{\circ} 28^{\prime} S-70^{\circ} 38^{\prime} \mathrm{O}\right)$ (Toro et al. 1999) y Chillán (36³4'S-7206’O) (González-Acuña et al. 
2004b) y en Zenaida auriculata Des Murs 1847 en esta última ciudad (González-Acuña et al. 2004a). El género Anomotaenia Cohn 1900 perteneciente a la familia Ciclophillidae Van Beneden in Braun 1900, fue aislado de L. maculipennis en el lago Puyehue por Torres et al. (1993).

Por otro lado, la especie Anomotaenia dominicanus Railliet \& Henry 1912 fue registrada en ejemplares de L. dominicanus capturados en los lagos Huillinco (Torres et al. 1991a), Yelcho, Tagua-Tagua (Torres et al. 1992), Llanquihue, Rupanco (4046'S$\left.72^{\circ} 30^{\prime} \mathrm{O}\right)$ y Caburga $\left(39^{\circ} 06 \mathrm{~S}-71^{\circ} 45^{\prime} \mathrm{O}\right.$ ) (Torres et al. 1993) y en L. maculipennis en el lago Villarrica (39¹3'S-7206'O) (Torres et al. 1993).

La familia Davaineidae Braun 1900 está representada por el género Raillietina Fuhrmann 1920 registrado por González-Acuña et al. (2004a) en Z. auriculata en la provincia de Ñuble (no se especifica lugar).

Dentro de la familia Diphillobothriidae Lühe 1910 se registró al género Diphyllobothrium Lühe 1910 en ejemplares de L. dominicanus provenientes del lago Calafquén $\left(39^{\circ} 30^{\prime} \mathrm{S}-72^{\circ} 11^{\prime} \mathrm{O}\right)$ (Figueroa et al. 1979) y estuario del río Valdivia (3952’S-73²0’O) (Torres et al. 1982) y en ejemplares de $L$. maculipennis también provenientes del lago Calafquén (Figueroa et al. 1980). Por último, González et al. (1980) lo reportan en aves del género Larus Linneus 1758 provenientes del lago Rupanco. La especie Diphillobothrium dendriticum Nitzsch 1824 fue obtenido por Torres et al. (1981) experimentalmente desde cinco ejemplares de $L$. dominicanus capturados en las costas de Mehuín $\left(39^{\circ} 25^{\prime} \mathrm{S}-73^{\circ} 11^{\prime} \mathrm{O}\right)$. Posteriormente, se ha identificado en infecciones naturales en $L$. dominicanus y en L. maculipennis en los lagos Puyehue, Rupanco, Caburga, Colico (3903'S7159’O), Villarrica, Llanquihue, Ranco, Maihue y Todos Los Santos $\left(41^{\circ} 05^{\prime} \mathrm{S}-72^{\circ} 15^{\prime} \mathrm{O}\right)$ (Torres et al. 1991b, 1993) (ver detalle por ave en Tabla I).

El primer registro dentro de la familia Hymenolepididae Ariola 1899, corresponde a Nybelin (1929), quien describe la especie Hymenolepis fernandensis Nybelin 1929 desde ejemplares de Turdus falcklandii Quoy \& Gaimard 1824 provenientes de la Isla Masatierra (33 $\left.42^{\prime} \mathrm{S}-79^{\circ} 01^{\prime} \mathrm{O}\right)$. Posteriormente, la especie Flamingolepis chileno Babero, Cattan \& Jensen 1981 es descrita desde dos ejemplares del flamenco andino Phoeni-coparrus andinus Philippi 1854 capturados en Laguna Lejía (233ㅇ'S-67²5'O) al norte de Chile (Babero et al. 1981). Por otra parte, el género Aploparaksis Clerc 1903 fue identificado en Podiceps major (Boddaert 1783) en los lagos Yelcho (Torres et al. 1992) y Ranco (Torres et al. 1993).

Por último, la especie Aploparaksis tinamoui Olsen 1970 fue registrada en la perdiz Nothoprocta perdicaria Kittlitz 1830 en la comuna de Nuble (Olsen 1970, Rubilar et al. 1996).

Dentro de la familia Paruterinidae Fuhrmann 1907, González-Acuña et al. (2000) identifican al género Anonchotaenia (Fuhrmann 1908) en ejemplares Callipepla californica Shaw 1798 capturadas en diferentes localidades de la provincia de Nuble.

La familia Tetrabothriidae Linton 1891 está representada por las especies Neotetrabothrius pellucidus Nybelin 1929 y Tetrabothrius lutzi Parona 1901 aislados en Spheniscus magellanicus Forster 1781. El género Tetrabothrius Rudolphi 1819 fue aislado en las aves P. major, Pelecanus thagus Molina 1782 desde el estuario del río Valdivia (Torres et al. 1982), en Larus scoresbii Traill 1823 en la costa Yaldad (Torres et al. 1991a), en $P$. brasilianus del lago Tagua-Tagua (Torres et al. 1992), en L. maculipennis de los lagos Tagua-Tagua (Torres et al. 1992) y Villarrica (Torres et al. 1993) y en Spheniscus humboldti Meyen 1834 en playas de la Quinta Región (entre $32^{\circ} 31^{\prime} \mathrm{S}-71^{\circ} 27^{\prime} \mathrm{O}$ y 3247'S-71³2'O) (Mann 1992). A nivel específico, Nybelin (1929) desde la Isla Masatierra registra las especies Tetrabothrius gracilis Nybelin 1916 en Fulmarus glacialoides (Smith 1840), Tetrabothrius gracilioides Nybelin 1929 en Diomedea melanophrys Temmink 1828, Tetrabothrius laccocephalus Spätlich 1909 y Tetrabothrius procerus Spätlich 1909 en Puffinus creatopus Coues 1868. Posteriormente, Housse (1945) cita a T. lutzi como causante de adelgazamiento y muerte en $S$. magellanicus. Por otro lado, la especie Tetrabothrius cylindraceus Rudolphi 1819 es identificada en ejemplares de L. dominicanus capturados en la costa de Yaldad y los lagos Huillinco (Torres et al. 1991a), Yelcho, Tagua-Tagua (Torres et al. 1992), Llanquihue, Rupanco y Caburga (Torres et al. 1993).

Clase Nematoda Potts 1932

En 17 especies de aves se han identificado un total de nueve familias de nematodos, 15 géneros y 18 a nivel específico. 
Como representantes de la familia Acuariidae Raillet, Hernry \& Sisoff 1912, el género Cosmocephalus Molina, 1858 es identificado por Mann (1992) en un ejemplar de S. humboldti de la Quinta Región. La especie Cosmocephalus obvelatus Creplin 1825 es identificada desde el esófago de dos ejemplares de Eudyptes crestatus Millar 1784 muertos en el Zoológico Maruyama en Sapporo ( $\left.141^{\circ} 12^{\prime} \mathrm{E}-43^{\circ} 03^{\prime} \mathrm{N}\right)$, Japón. Las aves provenían de Chile (no se especifica el lugar de captura) (Azuma et al. 1988). Posteriormente, Toro et al. (1999) y González-Acuña et al. (2000) reportan un 2 y $4 \%$ de prevalencia del helminto Dispharinx nasuta Rudolphi 1819 (syn.: D. spiralis) en ejemplares de C. livia y C. californica respectivamente. Este parásito afecta principalmente al orden Galliformes y se ubica en las paredes del proventrículo, produciendo hipertrofia nodular, úlceras y necrosis del epitelio (González-Acuña et al. 2000). Por último, la especie Gongylonema ingluvicola Ransom 1904 se aisló en un 2\% de los ejemplares de C. livia capturados en Santiago (Toro et al. 1999).

Perteneciente a la familia Acuarioidae Ditwood \& Wehr 1934 se identificaron estados larvales del género Skrjabinoclava Sobolev 1943 y adultos de la especie Skrjabinoclava tupacincai Wong \& Anderson 1988 en ejemplares de Calidris alba (Pallas 1764) capturadas en Antofagasta y Tongoy (30¹5'S-71 $\left.35^{\circ} \mathrm{O}\right)$ (Wong \& Anderson 1990). Los miembros de este género parasitan el proventrículo y ocasionalmente la mucosa del estómago. Un año más tarde, estos mismos autores y en la misma ave publican el registro de la especie Stellocaronema skrjabini Gilbert 1930 en Tongoy (Wong \& Anderson 1991).

La familia Anisakidae Skrjabin \& Karokhin 1945 está representada en Chile por el género Anisakis Dujardin 1845 reportado en dos ejemplares de $P$. thagus capturados en las costas entre Lebu (36 $33^{\circ}$ 'S-73 $\left.56^{\circ} \mathrm{O}\right)$ y Dichato $\left(37^{\circ} 37^{\prime} \mathrm{S}-73^{\circ} 41^{\prime} \mathrm{O}\right)$ (George-Nascimento \& Carvajal 1980). Posteriormente, Torres et al. (1982) registran el género Contracaecum Railliet \& Henry 1912 en ejemplares de $P$. thagus capturados desde el estuario del río Valdivia y en L. dominicanus provenientes de los lagos Todos Los Santos y Llanquihue (Torres et al. 1993). A su vez, Mann (1992) aísla este género desde el estómago e intestino de 12 ejemplares de S. humboldti provenientes de las playas de la Quinta Región. Por otro lado, la especie Contracaecum rudolphii Hartwich 1964 nematodo de amplia distribución geográfica, es identificado en L. maculipennis (Torres et al. 1983a, 1992), L. serranus, L. dominicanus (Torres et al. 1983a), P. major, P. atriceps (Torres et al. 1992) y en P. brasilianus (Torres et al. 1982, 1983a, 1991a, 1992, 1993, 2000), ave que por su distribución probablemente tenga un rol importante en la dispersión del parásito entre hemisferios (Torres et al. 1983b). Por último, la especie Porrocaecum depressum Zeder 1800 fue aislada en ejemplares de Milvago chimango Viellot 1816 en Chillán (San Martín 2004).

Perteneciente a la familia Ascaridiidae Blanchard 1849 se ha registrado la especie Ascaridia columbae Gmelin 1780 en ejemplares de C. livia en Santiago (Toro et al. 1999) y Chillán (González-Acuña et al. 2004b).

La familia Capillaridae Raillet 1915 está representada por el género Capillaria Zeder 1800, el cual ha sido aislado en $P$. major capturadas en el estuario del río Valdivia (Torres et al. 1982), en ejemplares de L. dominicanus provenientes de los lagos Huillinco (Torres et al. 1991a), Yelcho (Torres et al.1992), Caburga, Llanquihue (Torres et al. 1993) y el estuario del río Valdivia (Torres et al. 1982), en $L$. maculipennis en los lagos Llanquihue, Puyehue (Torres et al. 1993) y Yelcho (Torres et al. 1992) y C. livia provenientes de Chillán (GonzálezAcuña et al. 2004b).

La especie Capillaria caudinflata Molina 1858 fue aislada desde el duodeno e ileon de ejemplares de $N$. perdicaria (Rubilar et al. 1996), $C$. californica (González-Acuña et al. 2000) y $C$. livia (González-Acuña et al. 2004b) desde la provincia de Ñuble. Este parásito presenta un ciclo indirecto, siendo las lombrices de tierra sus hospedadores intermediarios. Se considera la presencia de este verme de gran importancia en aves de caza, pues puede ser responsable de importantes pérdidas cuando la carga es alta (Rubilar et al. 1996).

En 100 ejemplares de C. livia capturados en Santiago, un $1 \%$ presentó $C$. annulata Molina 1858 y $C$. obsignata Madsen 1945 y un $11 \%$ a $C$. columbae Madsen 1945 (Toro et al. 1999). Posteriormente, González-Acuña et al. (2004b) 
confirman la presencia de C. columbae en ejemplares de C. livia de Chillán. Por último, la especie Capillaria tenuissima (Rud. 1809) Yamaguti 1941 fue identificada en ejemplares de $M$. chimango de Chillán (San Martín 2004).

El único registro en la familia Habronematidae (Chitwood \& Wehr 1932) corresponde a la especie Cyrnea spinosa Gendre 1923 en M. chimango (San Martín 2004).

Los reportes pertenecientes a nemátodos de la familia Heterakidae Railliet \& Henry 1914 se han realizado en su mayoría en la provincia de Ñuble. Rubilar et al. (1996) registran a Subulura suctoria Molina 1860 en los ciegos de $N$. perdicaria con una intensidad de 3 a 307. La presencia de este nemátodo se relacionó con la presencia de pequeños escarabajos que actuarían como hospedadores intermediarios al ser consumidos por el ave. En los ciegos de C. californica (González-Acuña et al. 2000) y de Z. auriculata (González-Acuña et al. 2004a) se identificó la especie Heterakis gallinarum Schrank1788 parásito de ciclo directo, aunque también se ha considerado que la lombriz de tierra y algunos insectos pueden comportarse como hospedadores intermediarios. Se requieren infecciones severas de este parásito para producir lesiones de importancia patológica en el ave (González-Acuña et al. 2000).

Por último, González-Acuña et al. (2005) en la comuna de Río Verde (52³4’07S$71^{\circ} 32^{\prime} 440$ ) en la provincia de Magallanes, registran en el $100 \%$ de los ejemplares de Chloephaga picta Gmelin 1789 analizados al nemátodo H. dispar (Schrank 1790) con un rango en la intensidad de 1 a 1263 .

El único representante de la familia Tetrameridae Travassos 1914 corresponde al género Tetrameres Creplin 1846 identificado en ejemplares de C. livia en Santiago (Toro et al. 1999) y Chillán (Baier 2003, GonzálezAcuña et al. 2004b).

Perteneciente a la familia Trichostrongylidae Leiper 1908, se registró desde el Santuario Río Cruces ( $\left.39^{\circ} 37^{\prime} \mathrm{S} 73^{\circ} 07^{\prime} \mathrm{O}\right)$ al parásito estomacal Amidostomum anseris Zeder 1800 desde un ejemplar de Cygnus melancoryphus Molina 1782, quien además evidenció problemas de calcificación en su esqueleto (Schlatter et al. 1991).
Clase Trematoda Rudolphi 1808.

En ocho especies de aves se han identificado un total de cuatro familias de trematodos, siete géneros y sólo en cuatro ocasiones se ha llegado a nivel de especie.

Como representante de la familia Strigeidae Railliet 1919 ha sido identificada sólo la especie Apharyngostrigea pipientis (Faust 1918) desde ejemplares de la garza grande Ardea alba (Linneo 1758) provenientes desde Valparaíso ( $33^{\circ} 01^{\prime} \mathrm{S}$ 71³9’S) (Dubois 1981).

En la familia Echinostomatidae Looss 1899, Torres et al. (1982) reportaron el género Paryphostomum Dietz 1909 desde dos P. major capturadas en el estuario del río Valdivia. Por otro lado, la especie Stephanoprora denticulata (Rudolphi 1802) Odhner 1910 que presenta una baja especificidad de hospedador, fue aislada en las aves $P$. major del estuario del río Valdivia (Torres et al. 1982, 1983b) y lago Yelcho (Torres et al. 1992), en $P$. thagus del estuario del río Valdivia (Torres et al. 1982, 1983b), en $L$. dominicanus estuario del río Lingue $\left(39^{\circ} 15^{\prime} \mathrm{S}\right.$ $39^{\circ} 30^{\prime}$ O) (Torres et al. 1983b) y de los lagos Huillinco (Torres et al. 1991a), Yelcho, TaguaTagua (Torres et al. 1992), Llanquihue, Todos los Santos y Rupanco (Torres et al. 1993) y en $L$. maculipennis capturadas en el estuario del río Lingue (Torres et al. 1983b). Por otro lado, el género Echinostoma Rud, 1809 fue identificado en Z. auriculata en la provincia de Nuble (González-Acuña et al. 2004a).

Como representantes de la familia Diplostomidae Poirier 1886 ha sido identificada sólo la especie Diplostomum minutum Szidat 1964 desde ejemplares de L. dominicanus desde los lagos Villarrica y Caburga (Torres et al. 1993).

El único registro dentro la familia Heterophyidae Odhner 1914 corresponde al género Cryptocotyle Luhe 1899 aislado en las aves Ceryle torquata Linneus 1766 del lago Rupanco y en $L$. dominicanus en los lagos Llanquihue, Rupanco, Villarrica y Caburga (Torres et al. 1993).

Dentro de la familia Strigeidae Raillet 1919, Mann (1992) registra la especie Cardiocephalus physalis (Luzt 1914) (syn. Cardiocephaloides) en ejemplares de $S$. humboldti provenientes de playas de la Quinta Región de Chile. 


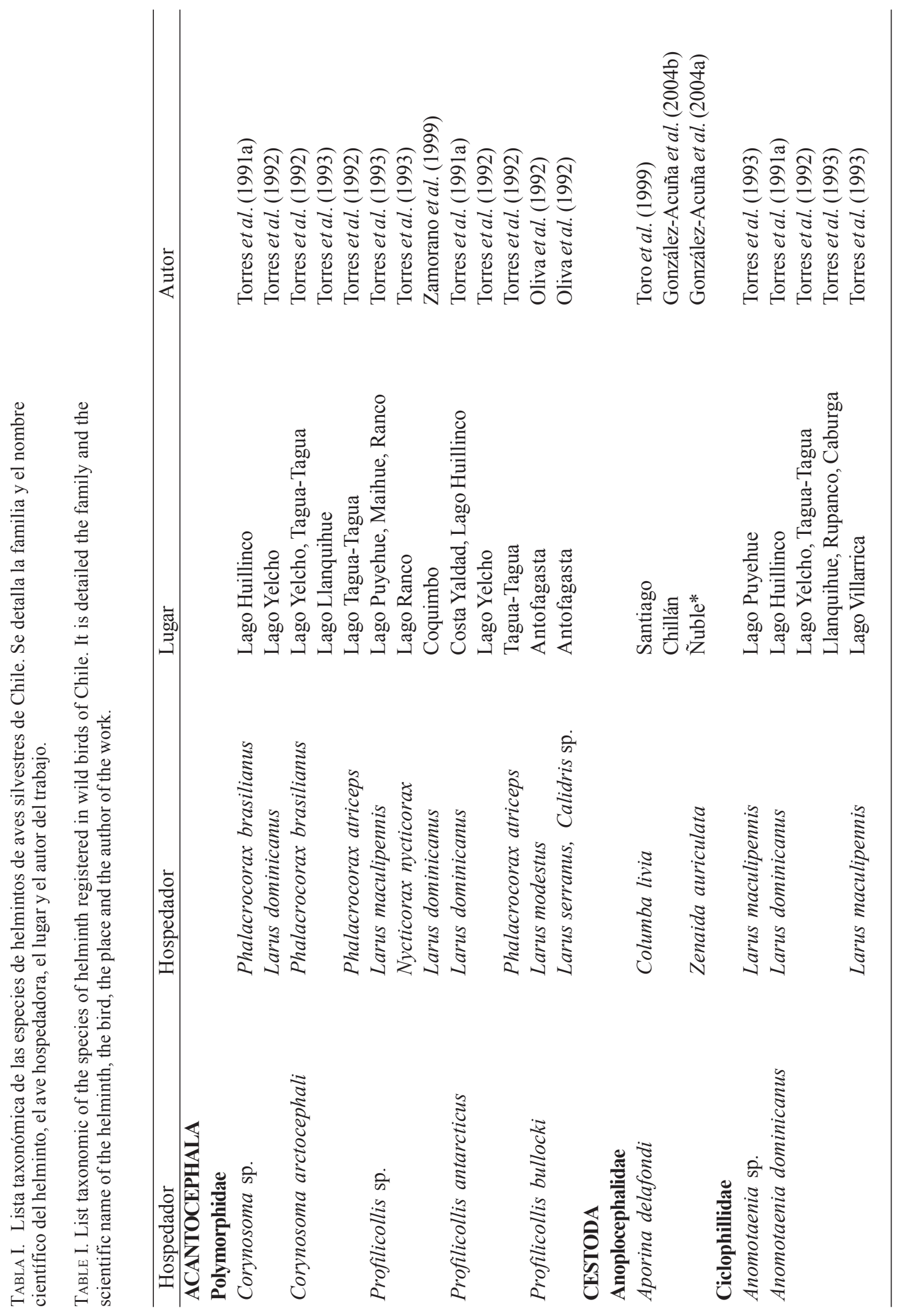


Estado del conocimiento de helmintos en aves silvestres: Hinojosa-SÁEz, A. \& D. GonZÁLeZ-Acuña

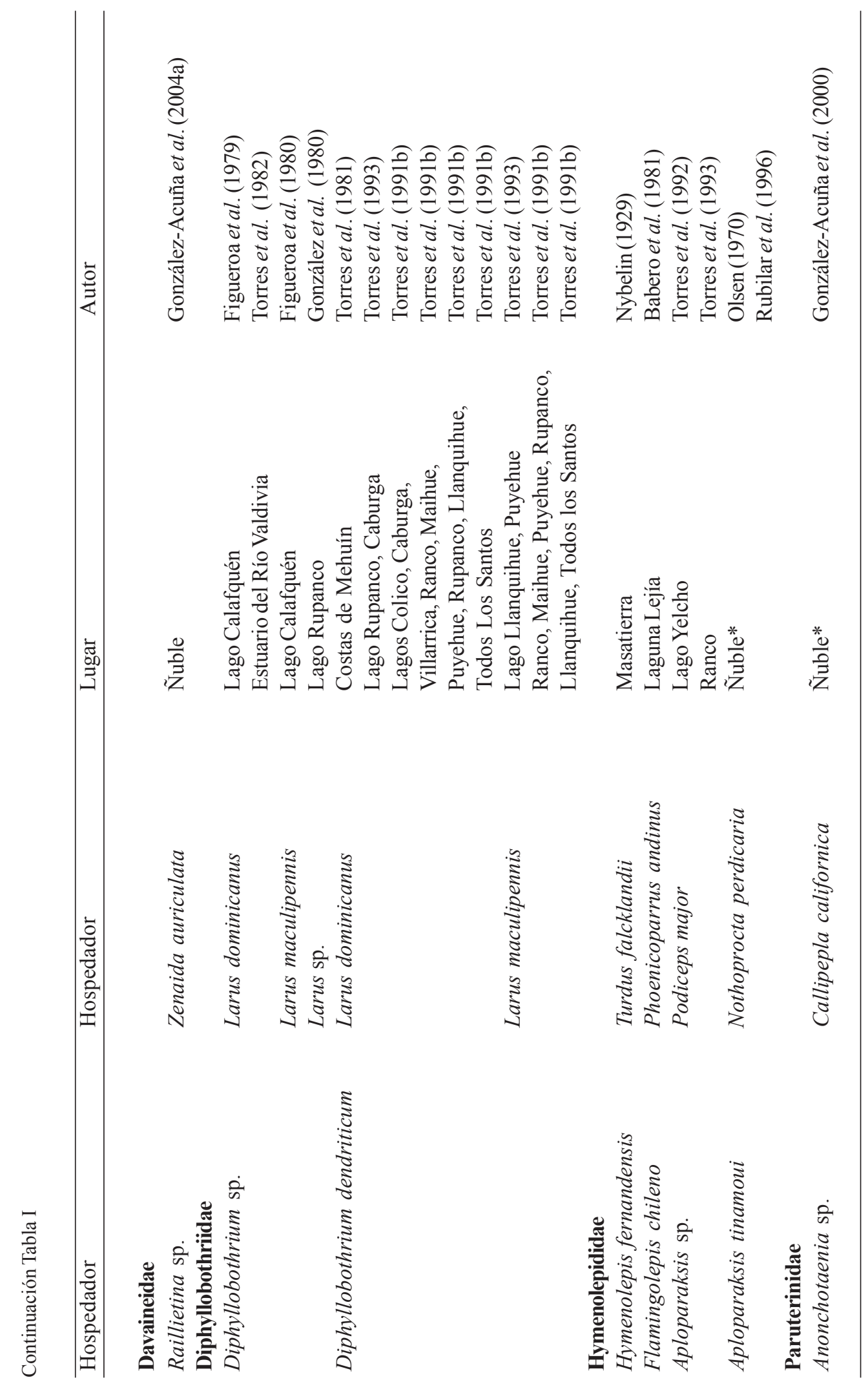


สิ

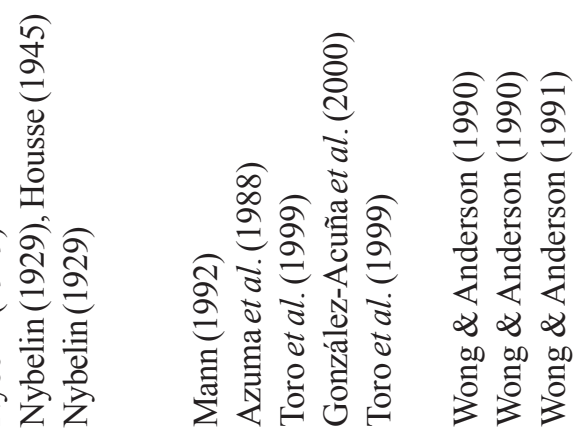

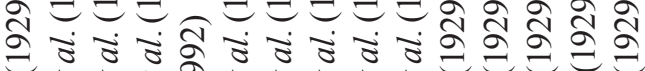

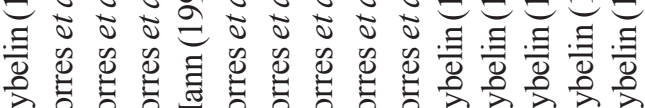

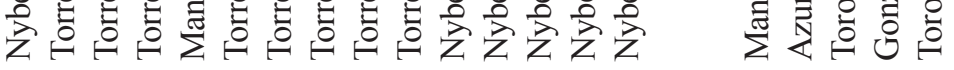

है

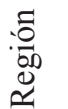

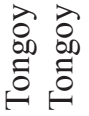

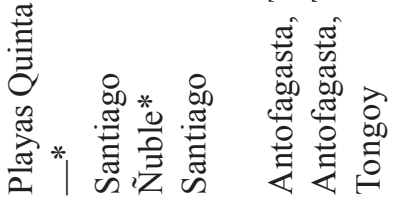

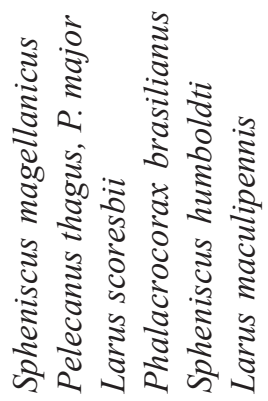
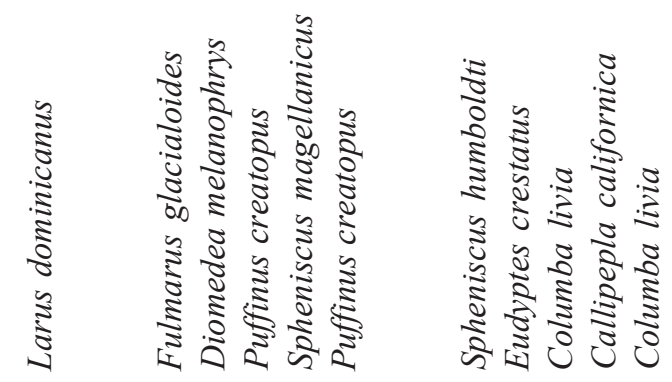

$\frac{2}{8} \sqrt{8}$ $\sqrt[3]{2}$

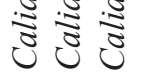

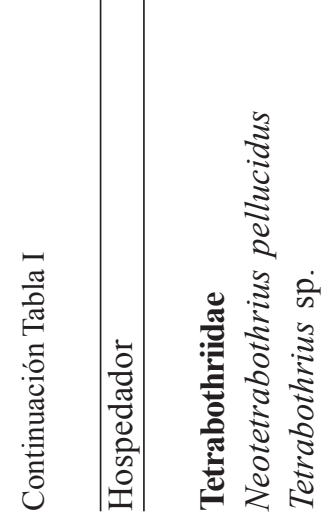

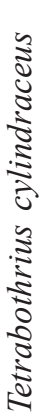
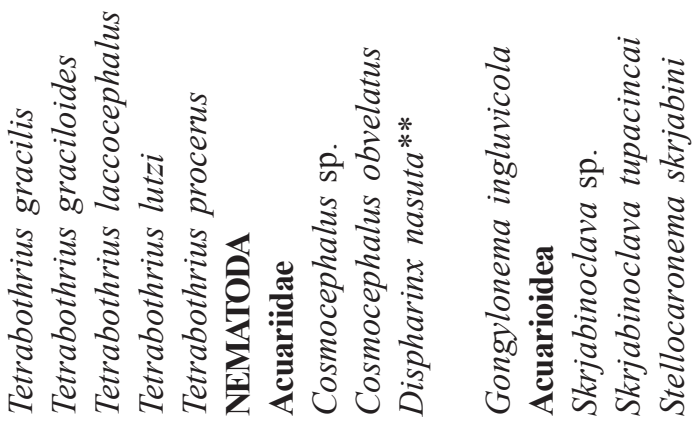
Estado del conocimiento de helmintos en aves silvestres: Hinojosa-SÁEZ, A. \& D. GonZÁlez-AcuÑa

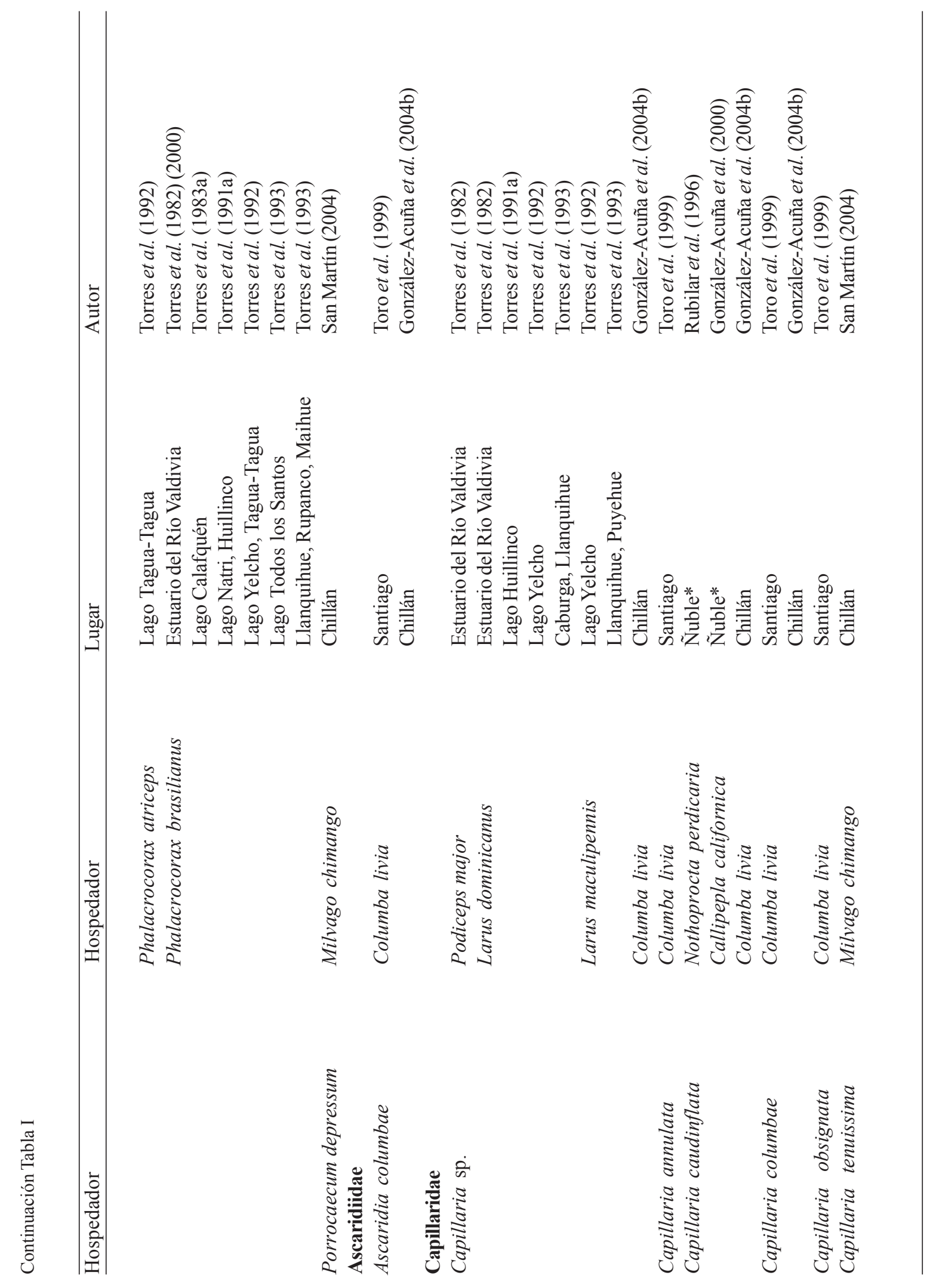


Gayana 69(2), 2005

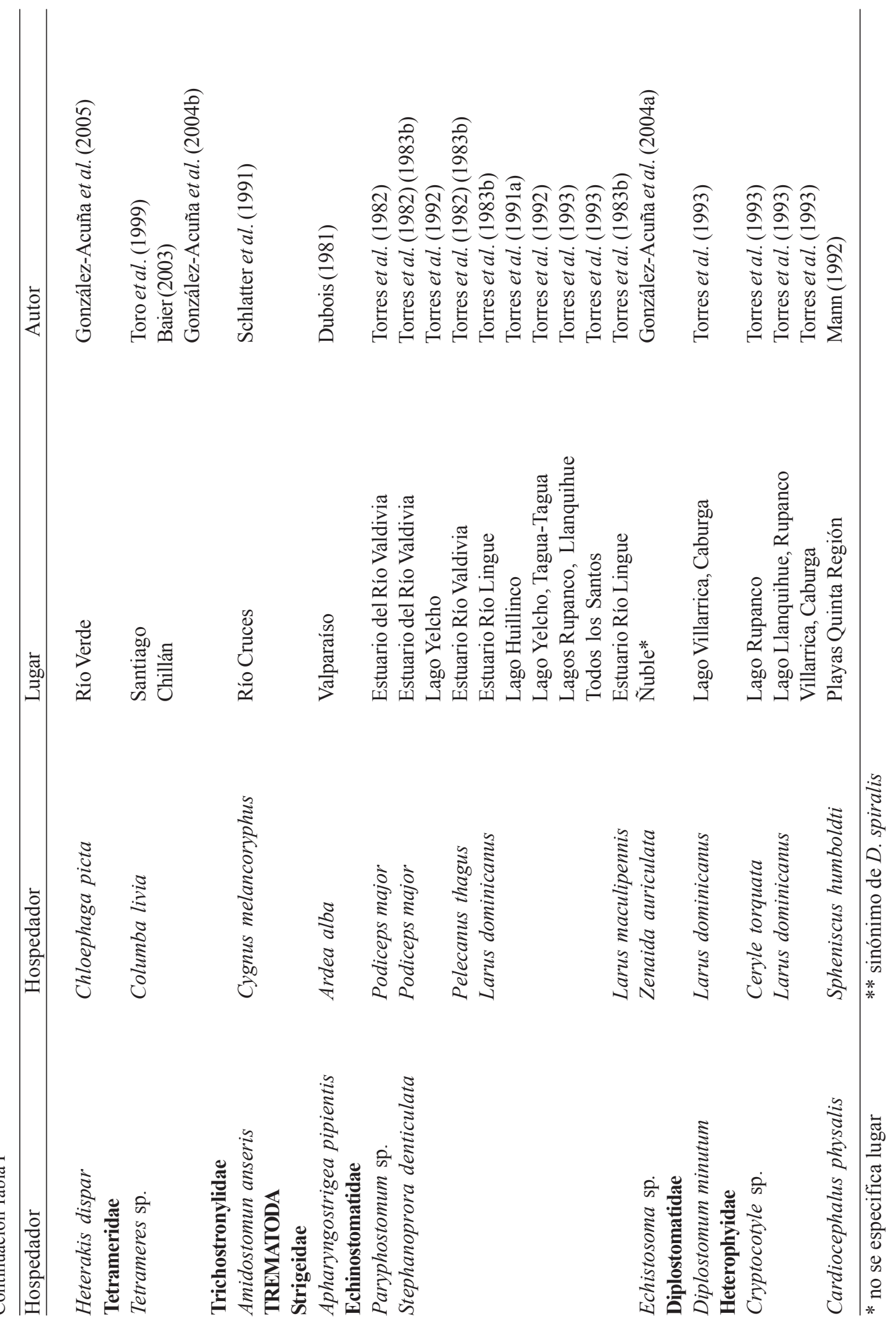




\section{DISCUSION}

Se han realizado un total de 33 trabajos en los cuales se reportan helmintos en aves silvestres en Chile. Los géneros de aves con mayores reportes corresponden a Larus (Charadriiformes) y Phalacrocorax (Pelecaniformes). Muchos de los helmintos registrados adquieren importancia por su potencial implicancia en salud pública y animal.

Un total de 30 especies distintas de aves han sido estudiadas, en las que se han identificado un total de 21 familias y 34 géneros diferentes de helmintos. Dentro de éstos, los géneros Tetrabothrius, Contracaecum y Capillaria son los más registrados.

Los trabajos más antiguos corresponden a Nybelin (1929) y Housse (1945) y la mayor cantidad de estudios se realizó en la década de los ochenta y noventa.

Si se divide los 76 años que han transcurrido entre el primer trabajo (1929) y el último (2005), por los trabajos realizados (33), dan cuenta de un total de 2,3 publicaciones por año, lo que evidencia una escasez de especialistas en el tema.

Los estudios abarcan desde Antofagasta por el norte, hasta la provincia de Magallanes por el sur, siendo la mayoría de los estudios efectuados en las ciudades de Valdivia, Chillán y Santiago, lo que se corresponden con la ubicación de centros de estudios superiores.

Con la presente revisión se complementa la lista parcial de helmintos para animales silvestres publicada por Cattan (1995). Cabe destacar que en el presente trabajo no se incluyó el trabajo de Fox et al. (1974), ya que el ave estudiada por este autor no provenía de Chile (com. per. Fox).

\section{AGRADECIMIENTOS}

Nuestros más sinceros agradecimientos al Dr. David Gibson, del Natural History Museum, de Londres; a los Doctores Patricio Torres y Roberto Schlatter, de la Universidad Austral de Chile, por su gran ayuda en la recopilación de material bibliográfico. A la Dra. Graciela Navone del CEPAVE (Centro de Estudios Parasitológicos y Vectores), Argentina, y al Dr. (C) Luis Balboa, de la Universidad Católica de Santiago, por sus aportes en la corrección del presente escrito. Y por último nuestros agradecimientos a la Dirección de Investigación de la Universidad de Concepción que ayudó a financiar parte de este trabajo.

\section{BIBLIOGRAFIA}

Azuma, H., M. Okamoto, M. Ohbayashi, Y. Nishine \& T. Mukai. 1988. Cosmocephalus obvelatus (Creplin 1825) (Nematoda: Acuariidae) collected from the esophagus of rockhopper penguin, Eudyptes crestatus. Japanesse Journal Veterinary Research 36: 73-77.

BAIER, P. 2003. Descripción de lesiones encontradas en palomas domésticas (Columba livia) de la ciudad de Chillán. Memoria de Título para optar al grado de Médico Veterinario. Facultad de Medicina Veterinaria. Universidad de Concepción, Chillán, Chile.

Babero, B., P. Cattan \& L. Jensen. 1981. Helmintofauna de Chile: IX. Flamingolepis chileno sp. n. parásito de Phoenicoparrus andinus Philippi. Boletín del Museo Nacional de Historia Natural de Chile 38: 105-109.

Brockerhoff A. \& L. Smales. 2002. Profilicollis novaezelandensis $\mathrm{n}$. sp. (Polymorphidae) and two other acanthocephalan parasites from shore birds (Haematopodidae and Scolopacidae) in New Zealand, with records of two species in intertidal crabs (Decapoda: Grapsidae and Ocypodidae). Systematic Parasitology 52: 55-65.

BRooK, D. 1985. Phylogenetics and the future of helminth systematics. Journal Parasitology 71: 719-727.

Cattan, P. 1995 Parte II: 15. Helmintos En: Diversidad Biológica de Chile. Comité Nacional de Diversidad Biológica. Artegrama. Santiago, Chile: $117-$ 126.

Chowdhury, N. \& Aguirre A. 2001. Helminth of wildlife science published. Enfield NH, USA. Plymouth UK. 513 pp.

Dubois, G. 1981. Notes Helminthologiques V: Strigaeidae Raillet, Diplostomatidae Porier et Proterodiplostomidae Dubois. Revue suisse Zool. 88: 227-232.

Figueroa, L., P. Torres, R. Schlatter, F. Asenjo, R. Franjola \& B. Contreras. 1979. Investigaciones sobre Pseudophyllidea (Carus 1813) en el sur de Chile III Estudio sobre Diphyllobothrium sp. en aves del lago Calafquén ( $\left.39^{\circ} 32^{\prime} \mathrm{S}-72^{\circ} 09^{\prime} \mathrm{O}\right)$. Boletín Chileno de Parasitología 34: 13-20.

Figueroa, L., P. Torres, R. Franjola \& R. Schlatter. 1980. Investigaciones sobre Pseudophyllidea (Carus, 1813) en el sur de Chile. VI. Infección por Diphyllobothrium (Cobbold) en Larus maculipennis Lichtenstein en el lago Calafquén. Boletín Chileno de Parasitología 35: 71-73.

Fox, J., S. Snyder, G. Schmidt \& L. Campbell. 1974. Infection with the nematode Streptocara incognita in the Chilean flamingo. Journal of Wildlife Diseases 10: 66-69.

George-Nascimento, M. \& J. Carvajal. 1980. Nuevos registros de nemátodos anisákidos en la fauna marina chilena. Boletín Chileno de Parasitología 35: 15-18.

González, H., V. Garrido, M. Landeta \& P. Martens. 1980. Nuevos aportes para la identificación de 
Diphyllobothrium sp., en el lago Rupanco, Chile. Boletín Chileno de Parasitología (35): 10-14.

González-Acuña D., O. Skewes-Ramm, L. RubilarContreras, A. Daugschies \& K. Pohlmeyer. 2000. Endoparásitos de codorniz (Callipepla californica) en Ñuble (Chile). Boletín Chileno de Ornitología 7: 23-25.

González-Acuña, D., A. Daugschies, L. Rubilar, K. Pohlmeyer, O. Skewes \& E. Mey. 2004a. Fauna parasitaria de la tórtola común (Zenaida auriculata de Murs 1847) (AVES: COLUMBIDAE) en Ñuble, Chile. Parasitología Latinoamericana. 59: 37-41. 2004.

GonzÁlez-Acuña, D., G. CAstillo, J. López, L. Moreno, S. Donoso, O. Skewes, R. Martínez \& J. Cabello. 2004b. Parásitos gastrointestinales y externos de la paloma doméstica (Columba livia) en la ciudad de Chillán, Chile. Agro-Ciencia 20(2): 107-112.

González-Acuña, D., O. Skewes, C. Candia, R. Palma, L. Moreno. 2005. Estudio del parasitismo gastrointestinal y externo en Caiquén Chloephaga picta Gmelin, 1789 (Aves, Anatidae) en la región de Magallanes, Chile. Parasitología Latinoamericana 60 (1-2): 86-89.

Housse, R. P. 1945. Las aves de Chile, su vida y sus costumbres. Editorial de la Universidad de Chile, Santiago, Chile. 390 pp.

Mann, A. 1992. Fauna parasitaria en el pingüino de Humboldt (Spheniscus humboldti), en la zona central de Chile. Memoria de Título para optar al grado de Médico Veterinario. Facultad de Medicina Veterinaria. Universidad de Chile. Santiago, Chile.

Mehlhorn, H. \& G. Piekarski. 1998. Grundriss der Parasitenkunde. Ed. Gustav Fisher. 5 auflage. 516 pp.

Monrrone, J. \& S. Coscarón. 1998. Biodiversidad de artrópodos argentinos. Ediciones Sur. La Plata, Argentina.

Nybelin, O. 1929. Säugetier und Vogelcestoden von Juan Fernandez. The Zoological Society of London. 27: 493-523.

Oliva, M., J. Luque \& A. Cevallos. 1992. Parásitos de Emerita analoga (Stimpson) (Crustacea): Implicaciones ecológicas. Boletín de Lima 79: 77-80.

Olsen, O. 1970. Aploparaksis tinamoui n. sp. cestode (Hymenolepididae) from the Chilean tinamou (Nothoprocta predicaria (Kittlitz, 1830) Tinaniformes). Revista Ibérica de Parasitología 30: 701-718.

Pérez-IÑigo, C. 1976. Parasitología. Especificidad Parasitaria. H. Blume, Ediciones. Madrid, España: 51-52.

Rubilar, L., E. Bertossi \& O. Skewes. 1996. Parasitismo gastrointestinal en la perdiz común (Nothoprocta perdicaria) en la zona de Nuble, Chile. Boletín Chileno de Parasitología 51: 35-37.

SAn Martín, J. 2004. Parasitismo gastrointestinal y externo en Tiuque Milvago chimango Viellot, 1816 (AVES: FALCONIDAE) en la zona de Nuble.
Memoria de Título para optar al grado de Médico Veterinario. Facultad de Medicina Veterinaria. Universidad de Concepción, Chillán, Chile.

Schlatter, R., J. Salazar, A. Villa \& J. Meza. 1991. Reproductive biology of black-necked Swans Cygnus melancoryphus at three Chilean wetland areas and feeding ecology at Rio Cruces. Wildfowlsuplement: 268-271.

Schmidt, G. \& L. Roberts. 1984. Fundamentos de parasitología. Principios y conceptos básicos. Editorial Continental, México: 15-38.

Tompkins, J. \& D. Clayton. 1999. Host resources govern the specificity of swift let lice: Size matters. Journal of Animal Ecology 68: 489-500.

Toro, H., C. Saucedo, C. Borie, R. Gouch \& H. Alcaino. 1999. Health status of free-living pigeons in the city of Santiago. Avian pathology 28: 619-623.

Torres, P., R. Franjola, L. Figueroa, R. Schlatter, H. GonzÁlez, B. Contreras \& R. Martin. 1981. Researches on Pseudophyllidea (Carus, 1813) in the south of Chile. IV Ocurrence of Diphyllobothrium dendriticum (Nitzch). Journal of Helminthology 55: 173-187.

Torres, P., L. Figueroa, A. SAldivia \& J. Barrientos. 1982. Gastrointestinal Heminths of Fish-Eating Birds from the Valdivia River, Chile. Journal Parasitology 68: 1157

Torres, P., V. Sierpe \& R. Schlatter. 1983a. Occurrence of Contracaecum rudolphii in New Host in Chile. Zeitchrift für Parasitenkunde 69: 397-399.

Torres, P., L. Figueroa \& A. Saldivia. 1983 b. Stephanoprora denticulata (Trematoda, Echinostomatidae) en gaviotas del sur de Chile. Boletín Chileno de Parasitología 38: 33-34.

Torres, P., E. Ruiz, W. Gesche \& A. Montefusco. 1991a. Gastrointestinal Helminths of Fish-Eating Birds from Chiloe Island, Chile. Journal of Wildlife Diseases. 27: 178-179.

Torres, P., V. Cubillos, W. Gesche, C. Rebolledo, A. Montefusco, C. Miranda, J. Arenas, A. Mira, M. Nilo \& C. Abello. 1991b. Difilobotriasis en salmónidos introducidos en lagos del sur de Chile: Aspectos patológicos, relación con infección humana, animales domésticos y aves piscívoras. Archivos de Medicina Veterinaria 23: 165-183.

Torres, P., A. Contreras, V. Cubillos, W. Gesche, A. Montefusco, C. Rebolledo, A. Mira, J. Arenas, J. Miranda, S. Asenjo \& R. Schlatter. 1992. Parasitismo en peces, aves piscívoras y comunidades humanas ribereñas de los lagos Yelcho y Tagua-Tagua, X Región de Chile. Archivos de Medicina Veterinaria 24: 77-91.

Torres, P., R. Schlatter, A. Montefusco, W. Gesche, E. Ruiz \& A. Contreras. 1993. Helminth parasites of piscivorous birds from lakes in the south of Chile. Memorias Instituto Oswaldo Cruz, Rio de Janeiro 88: 341-343.

Torres, P., J. Valdivieso, R. Schlatter, A. Montefusco, J. Revenga, F. Marín, J. Lamilla \& G. Ramallo. 2000. Infection by Contracaecum rudolphii 
Estado del conocimiento de helmintos en aves silvestres: HinojosA-SÁEZ, A. \& D. GonZÁlez-AcuÑa

(Nematoda: Anisakidae) in the Neotropic cormorant Phalacrocorax brasilianus, and fishes from the estuary of the Valdivia river, Chile. Studies Neotropical, Fauna \& Environment 35: 101-108.

Wong, P. \& R. ANDERSON. 1990. Host and geographic distribution of Skrjabinoclava spp. (Nematoda: Acuarioidea) in Nearctic shorebirds (Aves: Charadriiformes), and evidence for transmission in marine habitats in staging and wintering areas. Canadian Journal Zoology 68: 2539-2552.
Wong, P. \& R. ANDERson. 1991. Distribution of gizzard nematodes (Habronematoidea, Acuarioidea) of New World shorebirds (Charadriiformes), with special reference to localities of transmission. Canadian Journal Zoology 69: 2579-2588.

Zamorano, P., M. González, S. Garthe \& G. LunaJorquera. 1999. Endoparásitos de Larus dominicanus en la IV Región de Coquimbo. VI Congreso Chileno de Ornitología: 38. Antofagasta 11 al 13 de noviembre.

Fecha de recepción: 20/12/04

Fecha de aceptación: 10/05/05 